\title{
Percepción del estrés en estudiantes de odontología ante procedimientos nuevos
}

\section{Perception of stress in dental students in the face of new procedures}

\author{
Antonio Díaz-Caballero \\ Universidad de Cartagena, Cartagena, Colombia \\ José Bustillo-Arrieta \\ Universidad de Cartagena, Cartagena, Colombia \\ Clara Vergara-Hernández \\ Universidad de Cartagena, Cartagena, Colombia \\ Jhon Casseres-Navarro (D) \\ Universidad de Cartagena, Cartagena, Colombia \\ Alejandro Galvis-Franco \\ Universidad de Cartagena, Cartagena, Colombia
}

Open Access:

ISSN: $0124-2121$

E-ISSN: $2665-2420$

ARTÍCULO DE INVESTIGACIÓN CIENTÍFICA Copyright $\subset$ By Educación y Humanismo

Editor: Dhayana Fernández Matos

Correspondencia: cvergarah@unicartagena.edu .co

Recibido: $05-01-2021$ Aceptado: $10-08-2021$ En línea desde: 11-11-2021
Objective: to identify the parameters that affect the level of stress in dental students before preclinical and clinical practices. Method: a descriptive study that sought to establish the relationship of measurement variables in a sample of 150 students in the 2017-2018 period, with a participation of $30.6 \%$ of students from $4-5$ semester and $69.4 \%$ of students belonging to the clinical component of 6-9 semester. Results: The concept of academic stress was defined and characterized from the psychological approach. Regarding the stressors of the basic and clinical component, it was evidenced that $46.6 \%$ "sometimes" has had difficulty learning the theory and, when going into practice, lacks the required knowledge in preclinical and clinical, in addition that $44.66 \%$ "almost always" argue that the lack of time to carry out the practice of new procedures leads to stressors. Discussion and conclusion: Stress in the face of new procedures can be associated with several factors, mainly due to the lack of time for the study and practice of certain dental activities that will later affect daily clinical practice.

Keywords: Stress, fear, students, dentistry, health (DECS-BIREME)

Cómo citar este artículo (APA): Díaz-Caballero, A., Bustillo-Arrieta, J., Vergara-Hernández, C., Casseres-Navarro, J. y Galvis-Franco, A. (2021). Percepción del estrés en estudiantes de odontología ante procedimientos nuevos. Educación y Humanismo, 23(41), 313-326. https://doi.org/10.17081/eduhum.23.41.3772 


\section{Introducción}

La carrera de odontología requiere el desarrollo y aptitudes que someten al estudiante a una preparación exigente durante el proceso de pregrado, lo que conlleva a una mayor carga de ansiedad. El estrés en los estudiantes de odontología, de acuerdo al estudio de Evaristo-Chiyong y Chein-Villacampa (2015), puede ser multifactorial, por la responsabilidad por cumplir los horarios académicos, exceso de tareas y trabajos de cursos, evaluación de los profesores en el área clínica, tiempo para cumplir con las actividades; fomentando trastorno en el sueño, agotamiento, cefaleas, bajo rendimiento académico, entre otros. Es por ello que Misrachi-Launert et al. (2015) recomienda que se concientice que esta realidad pone en peligro la salud de los estudiantes de odontología, repercutiendo directamente en la salud de la población atendida en la preclínica y clínica universitaria.

El estrés según Sierra et al. (2003) se conceptualiza como una reacción de activación fisiológica, emocional, cognitiva y conductual ante estímulos y acontecimientos, en su conjunto esto se suministra por un exceso de actividades continuas. Desde el punto de vista biológico, Flórez (2000) lo precisa como un esquema adaptativo, y establece un componente de defensa, surgido para permitir al individuo responder ante situaciones adversas, en ese sentido es normal y comúnmente establecido para la persona y para su familia.

Desde la perspectiva psicológica, Pérez y Cartes (2015), lo asume como un estado afectivo, emocional, necesario para la adecuada adaptación del cuerpo al contexto, que estimula angustia en la persona. Y desde lo social y cultural, Bancalari y Oliva (2012), aduce que el estrés puede hacer parte del carácter del sujeto o de su entorno social.

También se considera estrés a una acción que se vaya a realizar por primera vez teniendo esa sensación de no contar con suficiente preparación o experiencia para realizarlo, un ejemplo claro es lo que se vive en la carrera de odontología donde en el plan de estudio se requiere una gama de actividades prácticas que suponen enfrentamiento con diferentes tipos de personalidades y ejecución de tratamientos que satisfagan las necesidades de los pacientes, lo anterior genera ansiedad y estrés frente a lo desconocido.

Varios autores (Jaramillo et al. 2008; Arrieta et al. 2015; Vergara y Navas 2017), se han dado a la tarea de investigar y mostrar preocupación por la salud mental de los estudiantes universitarios, llegando a la conclusión que este conjunto de actividades académicas si influye en su bienestar tanto físico como psicológico generando estados depresivos. Es de vital importancia identificar el proceso de transformación de los estudiantes en su paso por la preclínica y su capacidad de adaptarse al ciclo clínico en donde se evidencian temores, inseguridades y dificultades para enfrentar sus propios pacientes con diferentes formas de ser y comportamientos a los cuales no les fue preparado desde la teoría. 
El objetivo del estudio fue identificar los parámetros que afectan el nivel de estrés en los estudiantes de odontología ante nuevas prácticas teóricas y clínicas. Es necesario conocer cuáles fueron las situaciones que enfrentan los estudiantes en su diario vivir para poder establecer cuál de ellas genera dificultades y lograr canalizar esta respuesta de ansiedad hacia la mejora continua que beneficie la atención brindada en las facultades de odontología.

\section{Materiales y métodos}

Se efectuó un estudio de tipo descriptivo, transversal con enfoque cuantitativo, longitudinal. Este estudio posee un diseño de investigación cuantitativa de tipo no experimental, el cual, como describe Hernández, Fernández y Baptista (2010) es un "Estudio que se realiza sin la manipulación deliberada de variables y en el que sólo se observan los fenómenos en su ambiente natural para después analizarlos" (p. 149).

El presente estudio describe las diferentes percepciones que se tienen del estrés ante procedimientos nuevos en estudiantes del programa de Odontología de la Universidad de Cartagena. La población o universo de estudio fueron a estudiantes mencionados en forma no aleatoria desde cuarto a noveno semestre, identificados a través de un listado proporcionado por los archivos de la facultad de Odontología. El cálculo del tamaño de la muestra determinó en 150 el número de participantes, asumiendo un 95\% de confianza y un error del 5\%. El muestreo fue de tipo aleatorio estratificado por semestre, como el número de estudiantes por semestre es diferente, se tuvieron en cuenta las proporciones correspondientes al tamaño muestral, esto para evitar el sesgo de selección.

Se estudiaron variables como la distribución de los egresados según las características sociodemográficas, estrato y semestre cursado.

Para poder pertenecer al grupo de estudio debían ser estudiantes de pregrado de la Facultad de Odontología de la Universidad de Cartagena que quisieran participar en el estudio y estar matriculados en el momento de realizar las encuestas, que estuvieran comprometidos a participar en el estudio con las firmas de consentimiento informado. Se excluyeron los estudiantes menores de edad, que no firmaron el consentimiento informado o que estuvieran matriculados con más del $40 \%$ de inasistencia.

\section{Instrumentos}

Se realizaron encuestas tomadas de Willis y Lessler 1999; Ste, 2013 y de Barraza, 2006, 
disponibles en internet, las cuales fueron validadas a través de una prueba piloto con 50 estudiantes de los semestres descritos anteriormente.

Las preguntas fueron con escala tipo Likert con opciones de siempre, casi siempre, algunas veces, casi nunca y nunca, los cuales tendrían un valor de cinco a cero respectivamente obteniendo así los niveles de percepción para cada uno de los factores evaluados.

La recolección de datos se hizo en el transcurso del segundo periodo 2017-1 y 2018 en los estudiantes que fueron escogidos de acuerdo al semestre cursado de cuarto a noveno, y que se escogieron de manera aleatoria, los cuestionarios empleados llevan por nombre "formas de abordar el aprendizaje "en su versión ajustada r-spq-2f (Willis y Lessler 1999; Ste, 2013) y el Inventario Sisco del estrés académico (Barraza, 2006).

Una vez recolectadas las encuestas los datos fueron tabulados por intermedio de tabla matriz en Microsoft Excel. Para el procesamiento y análisis de la información se utilizó el programa Stata $x \circledR$ licencia número: 1990540768. El análisis descriptivo consistió en frecuencias y porcentajes de las variables sociodemográficas y la frecuencia de las variables de formas de estudio y estrés académico a través de un análisis univariado.

\section{Consideraciones éticas}

De acuerdo a la Resolución 8430 de 1993 del Ministerio de Protección Social de Colombia, este estudio se considera "sin riesgo" y la información recolectada es de carácter confidencial por lo que no se dará a conocer la identidad de los participantes respetando así su dignidad, la protección de sus derechos y su bienestar.

\section{Resultados}

La muestra estuvo constituida por un total de 150 estudiantes de Odontología de la Universidad de Cartagena, con un $56.66 \%$ correspondiente al sexo femenino y $43.33 \%$ perteneciente al sexo masculino y una edad promedio en la población de 21.36 años, la muestra cuenta con una participación del 30.6\% de estudiantes de $4-5$ semestre y $69.4 \%$ de estudiantes que pertenecen al componente de clínicas de 6-9 semestre, en cuanto al componente de variables sociodemográficas se encontró que la mayoría de la población encuestada pertenece al estrato nivel 2 en un $56.6 \%$ (Tabla 1 ). 


\section{Tabla 1.}

Variables sociodemográficas de los estudiantes

\begin{tabular}{lcc}
\hline Sexo & Variables & Frecuencias \\
\hline & & \\
\hline Masculino & & $65(43.33 \%)$ \\
\hline Femenino & & \\
\hline Semestre & 4 & \\
\hline & 5 & $21(14 \% .66 \%)$ \\
& 6 & $25(16.6 \%)$ \\
& 7 & $28(18.6 \%)$ \\
& 8 & $29(19.3 \%)$ \\
& 9 & $25(16.6 \%)$ \\
\hline Edad & 19 & $22(14.6 \%)$ \\
\hline & 20 & $20(13 \%)$ \\
& 21 & $31(20.6 \%)$ \\
& 22 & $28(18.6 \%)$ \\
& 23 & $26(17.3 \%)$ \\
24 & $35(23.3 \%)$ \\
\hline Estrato socioeconómico & $10(6.6 \%)$ \\
& 1 & $36(24 \%)$ \\
& 2 & $85(56.6 \%)$ \\
& 3 & $21(14 \%)$ \\
& 4 & $8(5.3 \%)$ \\
\hline
\end{tabular}

Fuente: Elaboración propia (2019)

Con respecto a los factores estresores del componente básico y clínico muestra que el $46.6 \%$ de la población encuestada manifiesta que algunas veces ha tenido dificultad para aprender la teoría y al pasar a la práctica carece de conocimientos requeridos en la preclínica y clínica.

Se observó que el 52\% de la población ha tenido dificultad de adaptación en la preclínica o clínica y el $50 \%$ ha tenido falta de confianza al aprender un procedimiento nuevo y realizarlo en la práctica clínica o preclínica.

Otros de los factores estresantes que se evidenció fue casi siempre la falta de tiempo para llevar a cabo la práctica de procedimientos nuevos con un $44.66 \%$, entre otros aspectos que se evaluaron se presentó que el $43.33 \%$ de los encuestados ha tenido falta de interés para aprender a fondo un procedimiento nuevo o tema que no es de su agrado. (Tabla2)

Tabla 2.

Variables relacionadas con factores estresores del componente básico y clínico Universidad de Cartagena

\begin{tabular}{llllll}
\hline Factores estresores & $\begin{array}{l}\text { Nunca- } \\
\text { frecuencia }\end{array}$ & $\begin{array}{l}\text { Casi nunca- } \\
\text { frecuencia }\end{array}$ & $\begin{array}{l}\text { Algunas } \\
\text { veces- } \\
\text { frecuencia }\end{array}$ & $\begin{array}{l}\text { Casi } \\
\text { siempre- } \\
\text { frecuencia }\end{array}$ & $\begin{array}{l}\text { Siempre- } \\
\text { frecuenc } \\
\text { ia }\end{array}$ \\
\hline
\end{tabular}




\begin{tabular}{|c|c|c|c|c|c|}
\hline $\begin{array}{l}\text { Ha tenido dificultad para aprender } \\
\text { la teoría y al pasar a la práctica } \\
\text { careces de conocimientos } \\
\text { requeridos en la preclínica y } \\
\text { clínica. }\end{array}$ & $20(13.33 \%)$ & $35(23.33 \%)$ & $70(46.66 \%)$ & $15(10 \%)$ & )$^{10(6.66 \%}$ \\
\hline $\begin{array}{l}\text { Ha tenido dificultad de adaptación } \\
\text { en la preclínica o clínica }\end{array}$ & $7(4.66 \%)$ & $13(8.66 \%)$ & $78(52 \%)$ & $37(24.66 \%)$ & $15(10 \%)$ \\
\hline $\begin{array}{l}\text { Ha tenido falta de confianza en ti } \\
\text { mismo al aprender un } \\
\text { procedimiento nuevo y realizarlo } \\
\text { en la práctica clínica o preclínica }\end{array}$ & $10(6.66 \%)$ & $20(13.33 \%)$ & $75(50 \%)$ & $25(16.66 \%)$ & $\begin{array}{l}20(13.33 \\
\%)\end{array}$ \\
\hline $\begin{array}{l}\text { Ha tenido falta de tiempo para } \\
\text { llevar a cabo la práctica de } \\
\text { conocimientos nuevos }\end{array}$ & $7(4.66 \%)$ & $12(8 \%)$ & $45(30 \%)$ & $67(44.66 \%)$ & $\begin{array}{l}19(12.66 \\
\%)\end{array}$ \\
\hline $\begin{array}{l}\text { Ha tenido suficiente tiempo de } \\
\text { práctica para el examen en el } \\
\text { laboratorio }\end{array}$ & $67(44.66 \%)$ & $26(17.33 \%)$ & $15(10 \%)$ & $35(23.33 \%)$ & $7(4.66 \%)$ \\
\hline $\begin{array}{l}\text { Ha presentado sobrecarga de } \\
\text { trabajos y prácticas académicas }\end{array}$ & $6(4 \%)$ & $3(2 \%)$ & $58(38.66 \%)$ & $53(35.33 \%)$ & $\begin{array}{l}20(13.33 \\
\%)\end{array}$ \\
\hline $\begin{array}{l}\text { Ha tenido suficiente tiempo de } \\
\text { descanso en el transcurso del } \\
\text { semestre }\end{array}$ & $36(24 \%)$ & $68(45.33 \%)$ & $20(13.33 \%)$ & $15(10 \%)$ & )$^{11(7.33 \%}$ \\
\hline $\begin{array}{l}\text { Cuenta con respaldo para } \\
\text { comprar los materiales necesarios } \\
\text { para la práctica de procedimientos } \\
\text { nuevos }\end{array}$ & $12(8 \%)$ & $37(24.66 \%)$ & $45(30 \%)$ & $40(26.66 \%)$ & $\begin{array}{l}16(10.66 \\
\%)\end{array}$ \\
\hline $\begin{array}{l}\text { Observa materiales de apoyo } \\
\text { como videos, audios etc. para la } \\
\text { práctica en laboratorio }\end{array}$ & $10(6.66 \%)$ & $25(16.66 \%)$ & $45(30 \%)$ & $56(37.33 \%)$ & $\begin{array}{l}\text { 14(37.33 } \\
\%)\end{array}$ \\
\hline $\begin{array}{l}\text { Ha presentado Falta de interés } \\
\text { ante un nuevo tema o } \\
\text { procedimiento }\end{array}$ & $35(23.33 \%)$ & $20(13.33 \%)$ & $65(43.33 \%)$ & $25(16.66 \%)$ & $5(3.33 \%)$ \\
\hline
\end{tabular}

Fuente: Elaboración propia (2019)

Respecto al cuestionario "formas de abordar el aprendizaje" se evidenció que el 52\% de los encuestados le parecen los temas nuevos interesantes algunas veces, además algunas veces el $47.33 \%$ dedican una parte del tiempo libre a buscar información sobre temas interesantes vistos en clase, también se evidenció que el $41.33 \%$ casi nunca les parece útil los temas en profundidad, ya que solo confunde y hace perder el tiempo.

Entre otros aspectos encontrados el 54\% de los encuestados prefieren no estudiar los temas que les parecen irrelevantes y que probablemente no va a entrar en la prueba. (Tabla 3). 
Tabla 3.

Encuesta percepción de estrés ante procedimientos nuevos

\begin{tabular}{|c|c|c|c|c|c|}
\hline & $\begin{array}{l}\text { Nunca- } \\
\text { frecuencia\% }\end{array}$ & $\begin{array}{l}\text { Casi nunca - } \\
\text { frecuencia\% }\end{array}$ & $\begin{array}{l}\text { Algunas } \\
\text { veces - } \\
\text { frecuencia\% }\end{array}$ & $\begin{array}{l}\text { Casi siempre } \\
\text { Frecuencia\% }\end{array}$ & $\begin{array}{l}\text { Siempre } \\
\text { Frecuencia\% }\end{array}$ \\
\hline $\begin{array}{l}\text { Estudió en serio la materia } \\
\text { que se pasa en clases o que } \\
\text { está en el programa del } \\
\text { curso }\end{array}$ & $0(0 \%)$ & $15(10 \%)$ & $42(28 \%)$ & $41(27.33 \%)$ & $52(34.66 \%)$ \\
\hline $\begin{array}{l}\text { La mayoría de los temas } \\
\text { nuevos me parecen } \\
\text { interesantes, y pasó tiempo } \\
\text { extra buscando más } \\
\text { información sobre ellos. }\end{array}$ & $3(2 \%)$ & $15(10 \%)$ & $79(52.66 \%)$ & $53(35.33 \%)$ & $0(0 \%)$ \\
\hline $\begin{array}{l}\text { Dedicó una buena parte de } \\
\text { mi tiempo libre a buscar } \\
\text { información sobre temas } \\
\text { interesantes que hemos } \\
\text { vistos en clases }\end{array}$ & $0(0 \%)$ & $16(10.66 \%)$ & $71(47.33 \%)$ & $48(32 \%)$ & $15(10 \%)$ \\
\hline $\begin{array}{l}\text { No me parece útil estudiar } \\
\text { los temas en profundidad. } \\
\text { Eso solo confunde y hace } \\
\text { perder el tiempo, cuando lo } \\
\text { único que se necesita es un } \\
\text { conocimiento general de } \\
\text { los temas }\end{array}$ & $35(23.33 \%)$ & $62(41.33 \%)$ & $49(30.66 \%)$ & $4(2.66 \%)$ & $0(0 \%)$ \\
\hline $\begin{array}{l}\text { "Es irrelevante estudiar el } \\
\text { material que } \\
\text { probablemente no va a } \\
\text { entrar en la prueba" }\end{array}$ & $0(0 \%)$ & $23(15.33 \%)$ & $81(54 \%)$ & $46(30.66 \%)$ & $0(0 \%)$ \\
\hline $\begin{array}{l}\text { Generalmente me limito a } \\
\text { estudiar solo lo que } \\
\text { establece el docente, } \\
\text { porque creo que es } \\
\text { innecesario hacer trabajo } \\
\text { extra }\end{array}$ & $14(9.33 \%)$ & $56(37.33 \%)$ & $65(43.33 \%)$ & $15(10 \%)$ & $0(0 \%)$ \\
\hline
\end{tabular}

Fuente: elaboración propia (2019)

\section{Discusión y conclusiones}

Se reportan en la literatura diversos estudios referentes a las situaciones de estrés que afrontan los estudiantes de odontología, no sólo en Colombia, si no a nivel mundial, ya que es una condición intrínseca referente a la carrera (Ortiz-Fernández et al. 2015; Collazo et al. 2011; Jaramillo et al. 2008).

En el análisis del test Sisco del estrés académico se encontró que para el 43,33\% de los encuestados, la falta de interés ante un nuevo tema o procedimiento al considerarlo irrelevante es un factor estresor, ya que podría este nuevo tema ser útil en algún punto de su vida profesional y no tendrían el conocimiento necesario para abordar casos clínicos; concordando con Bonilla et al. (2014) quien reportó que uno de los factores estresores para 
los estudiantes fue "no entender temas abordados", comprendiendo que este temor surge como respuesta a las consecuencias que implican el desconocimiento frente al ejercicio de la carrera.

Entre otros aspectos destacados en este estudio se menciona que el $30.66 \%$ de los encuestados referente al cuestionario titulado "formas para abordar el aprendizaje", "algunas veces" no les parece ventajoso profundizar los temas que les parecen irrelevantes, esto les confunde y hace perder el tiempo, también se observó que el $47.33 \%$ de los encuestados "algunas veces" dedican una gran parte del tiempo libre a buscar información sobre temas atractivos que han desarrollado en clases. De acuerdo a Arbildo-Vega (2014) y Díaz et al. (2017) los estudiantes de odontología deben saber identificar y manejar los factores estresantes que conlleva el transcurso de la carrera y, tener en cuenta que todos los temas vistos en clase son importantes y requieren atención fuera de la academia para reforzar todas las debilidades obtenidas. La elocuencia de estos autores debe ser estudiada por los estudiantes de la facultad de odontología de la Universidad de Cartagena para que abran su mente hacia los conocimientos que serán de gran ayuda en su futuro profesional.

Igualmente, se observó la falta de interés por parte del alumnado para aprender a fondo un tema o procedimiento nuevo que no sean de su agrado, especialmente cuando el contenido no tiene sentido para ellos, debido a que, no les hallan aplicación, por lo que no alcanza a ser aprendizajes significativos, por ello es beneficioso lograr que contenidos teóricos se articulen con los prácticos teniendo en cuenta los conocimientos adquiridos por los alumnos, con sus experiencias de vida, y así las temáticas afrontadas en el aula alcancen significado.

Por otra parte, se encontró que el 38,66\% de los encuestados presentó sobrecarga de trabajos y prácticas académicas, similar a lo reportado en un estudio realizado en la Universidad de Perú donde se afirmó que "la sobrecarga de trabajos" fue uno de los principales factores estresores. Sin embargo, en el presente estudio, el exceso de trabajos no fue el principal factor estresor.

Por otro lado, el tiempo es un factor importante en la vida Universitaria, el cual, debe ser bien distribuido y aprovechado, los espacios entre clases suelen ser empleados para adelantar deberes, sin embargo, los estudiantes de odontología de la Universidad de Cartagena, no cuentan con suficientes horas para completar sus actividades académicas, sus jornadas diarias ocupan en promedio 8 horas al día por lo que se encontró que un $44,66 \%$ de los encuestados "casi siempre" y "nunca" en igual porcentaje han tenido suficiente tiempo para poner en práctica los conocimientos adquiridos y mejorar su destreza mediante la práctica en laboratorios, datos que se asemejan al estudio antes mencionado de la Universidad de Perú sobre estrés y desempeño académico en estudiantes de odontología, donde se evidenció que el tiempo limitado para realizar prácticas de laboratorio se considera un factor estresor en los estudiantes de odontología de dicha institución. Igualmente, los resultados obtenidos en el presente trabajo coinciden con lo expresado por 
Flórez (2000), quienes afirman que las largas jornadas laborales entre otros aspectos influyen de manera importante en la ansiedad de los individuos. Águila et al. (2015), reportaron que los exámenes, la sobrecarga académica y la falta de tiempo para el descanso de las actividades académicas constituyen los mayores factores estresores, afirmando una vez más que el tiempo es indispensable para los estudiantes y no contar con las suficientes horas para desempeñar actividades académicas, propias y recreación es una fuente considerable de tensión y ansiedad.

Con lo anteriormente descrito, se hace necesario que dentro del trabajo pedagógico y educativo de docentes, se generen aulas que fomenten hábitos de estudio saludables, permitiendo a los alumnos ser más organizados con el tiempo requerido para completar compromisos académicos y personales, de esta manera se estarían formando profesionales más capacitados y responsables con su entorno, así mismo, autores como Díaz et al (2011) resaltan el rol constructivista que han asumido los programas de área de la salud, sin embargo, señalan que es necesario un cambio de paradigma de docentes y estudiantes, concertando con lo planteado por los autores del presente trabajo quienes buscan una mejora continua del personal odontológico.

Otro aspecto importante es la adaptación al entorno clínico, indispensable para el desempeño de labores prácticas en el campo de la salud, en la presente investigación se encontró que el 52 \% de los encuestados presentó dificultad de adaptación en la preclínica o clínica. Lo anterior genera frustración ante la imposibilidad de ejecutar los procesos de atención de manera adecuada. Vergara y Zaror (2008) aduce que el proceso formativo en las clínicas odontológicas se facilita en un contexto en el que el alumno aplica las ciencias básicas y preclínicas en este escenario clínico, con la necesidad de desenvolverse y capacitarse en competencias básicas y clínicas con el acompañamiento de un tutor que guíe su proceso.

Lazarus y Flokman (1984) explican mejor el proceso de adaptación al cambio con su teoría "conducta de afrontamiento" la cual se entiende como esfuerzos cognitivos y contextuales en proceso de cambio permanente, que se desarrollan para organizar las exigencias externas/internas de cada persona y que dependen de la diversidad de elementos resolutivos con que cuente el individuo. De esta manera, es necesario aplicar estrategias que faciliten el paso de actividades teóricas a clínicas y disminuir las reacciones de agobio presentadas por el alumnado de las distintas facultades de odontología.

El estrés constante en la vida de los estudiantes de Odontología de la Universidad de Cartagena puede generar impactos perjudiciales en su salud y calidad de vida, teoría reforzada por Díaz et al (2017), donde demuestran que los estudiantes al desarrollar sus compromisos académicos, adquieren prácticas posturales incorrectas, lo cual puede desencadenar dolor muscular de forma prematura, en zonas como el cuello, espalda, hombros y manos. Del mismo modo, Tamayo et al. (2015) reportaron que los alumnos de odontología a causa de la carga académica y el estrés adquieren hábitos alimenticios dañinos 
como: alimentación a deshoras, elección inadecuada de alimentos, inapetencia y algunas veces pueden pasar una jornada académica completa sin ingerir alimentos, factores que pueden desencadenar síndrome de intestino irritable y por ende el deterioro de la salud.

La carrera de odontología trae consigo ciertas necesidades adquisitivas de materiales e instrumental para el desarrollo de las prácticas, es importante contar con los recursos económicos para poder acceder a los implementos requeridos, sin embargo, en el presente estudio se evidenció que el $56,6 \%$ de los estudiantes pertenece al estrato 2 y sólo un $30 \%$ de ellos cuentan algunas veces con respaldo para comprar los materiales para la práctica de procedimientos nuevos, desencadenando estrés, así mismo Arrieta et al. (2015) en su estudio concuerda que los ingresos económicos bajo de dos salarios mínimos se relacionan a un bajo rendimiento académico y por consiguiente genera un factor desencadenante de estrés ya que el estudiante no va a tener los recursos necesarios para ejercer sus prácticas preclínicas y clínicas en la Facultad de Odontología. A su vez Feldman y Kubota (2015) opina que uno de los indicadores más importantes del éxito universitario corresponde al promedio de calificaciones el cual influye potencialmente en la ayuda financiera, disminuyendo de esta forma su ansiedad.

Luego de obtener los resultados de las encuestas y estudiar detalladamente cada uno de los factores estresores para los estudiantes de odontología, se debe plantear estrategias de acción que permitan disminuir el porcentaje de estrés generado por diferentes situaciones que son cotidianas en la vida estudiantil y que, por ende deberían lograr ser manejadas de la manera más positiva en favor de la salud física y mental del individuo, así como del buen desempeño durante el pregrado. Presta et al. (2006) y Ortiz-Fernández (2015) opinan que es importante saber manejar el estrés en su práctica día a día particular.

Los resultados de Vergara et al. (2017) aportan en la disminución de los factores estresores desde el significado que adquiere la "adaptación a la etapa clínica hasta cumplir el sueño de ser odontólogo, el cual es un proceso de identificar estilos de aprendizaje y hábitos de estudio que no existían, pero que han aprendido con el tiempo" (p. 300).

Se concluye que los factores estresores más predominantes fueron la falta de tiempo con un $44,66 \%$, dificultad de adaptación en la preclínica o clínica con un $52 \%$ y la falta de confianza en sí mismo en un $50 \%$, la sobrecarga académica genera estrés algunas veces y casi siempre en un $38,66 \%$ y $35,33 \%$ respectivamente. Es evidente que los porcentajes de estrés son elevados y desencadenan manifestaciones somáticas como somnolencia y problemas de digestión.

Finalmente se propone que en la inducción a las clínicas se incluyan "estrategias de afrontamiento y entrenamiento de habilidades de solución de problemas" con el propósito de mejorar el bienestar personal de los estudiantes de odontología, así como la calidad de su trabajo clínico. 


\section{Referencias}

Águila, B. A., Castillo, M. C., de la Guardia, R. M., \& Achon, Z. N. (2015). Estrés académico. Edumecentro, 7(2), 1-12.

Arbildo-Vega, H. I. (2015). Niveles de ansiedad en estudiantes de odontología de una universidad peruana. Revista KIRU, 11(2), 1-23.

Arrieta, K., Blanco, M., \& Cabrera, R. (2015). Relación entre satisfacción vocacional, estilos de afrontamiento y estrés percibido con el síndrome de burnout en estudiantes de odontología. [Tesis de grado, Universidad de Cartagena]. http://repositoriounicartagena.edu.co

Bancalari, S L., \& Oliva M, P. (2012). Efecto de la Musicoterapia sobre los Niveles de Estrés de los Usuarios Internos de la Clínica de Odontología de la Universidad del Desarrollo. International Journal of Odontostomatology, 6(2), 189-193. https://doi.org/10.4067/S0718-381X2012000200012

Barraza, A. (2006). Un modelo conceptual para el estudio del estrés académico. Revista electrónica de psicología iztacala, 9(3), 1-20.

Bonilla, M., Delgado, K., \& León, D. (2014). Nivel de estrés académico en estudiantes de odontología de una universidad del distrito de Chiclayo. [Doctoral dissertation, Tesis de pregrado, Universidad Católica Santo Toribio de Mogrovejo]. https://tesis.usat.edu.pe/handle/20.500.12423/309

Collazo, M., Seoane, M., \& Hernández, O. (2011). Perfil sociodemográfico y desempeños de los estudiantes de la carrera de Odontología (UdelaR). Odontoestomatología, 13(18), 46-55.

Díaz, S., Arrieta, K., \& Guette, A. (2011). Problemas de salud y calidad de vida en estudiantes de odontología. Universidad y Salud, 19(1), 51. https://doi.org/10.22267/rus.171901.68

Díaz, S., Martínez Redondo, M., Zapata Teherán, A. M. (2017). Academic performance and quality of life related to health in dentistry students. Salud Uninorte, 33(2), 139-151. https://doi.org/10.14482/sun.33.2.10540

Evaristo-Chiyong, T., \& Chein-Villacampa, S. (2015). Estrés y desempeño académico en estudiantes de Odontología. Odontología Sanmarquina, 18(1), 23. https://doi.org/10.15381/os.v18i1.11336

Feldman, D. B., \& Kubota, M. (2015). Hope, self-efficacy, optimism, and academic achievement: Distinguishing constructs and levels of specificity in predicting college grade-point average. Learning and Individual Differences, (37), 210-216. 


\section{https://doi.org/10.1016/j.lindif.2014.11.022}

Flórez-Alarcón, L. (2000). Análisis del estrés diario en el personal de odontólogos, auxiliares y operadoras de una clínica odontopediátrica. Psicología conductual, (8), 375-388.

Folkman, S., \& Lazarus, R. S. (1984). Stress, appraisal, and coping. Springer Publishing Company.

Frese, C., Wolff, D., Saure, D., Staehle, H. J., \& Schulte, A. (2018). Psychosocial impact, perceived stress and learning effect in undergraduate dental students during transition from pre-clinical to clinical education. European Journal of Dental Education, 22(3), e555-e563. https://doi.org/10.1111/eje.12352

González, L. F., Hernández, A. G., \& Torres, M. T. (2017). Relaciones entre estrés académico, apoyo social, optimismo-pesimismo y autoestima en estudiantes universitarios. Electronic Journal of Research in Education Psychology, 13(35), 111130.

Halboub, E., Nasser Alhajj, M., Mohammed AlKhairat, A., Madani Sahaqi, A.-A., \& Faeq Ali Quadri, M. (2018). Perceived Stress among Undergraduate Dental Students in Relation to Gender, Clinical Training and Academic Performance. Acta Stomatologica Croatica, 52(1), 37-45. https://doi.org/10.15644/asc52/1/6

Harris, M., Wilson, J. C., Hughes, S., \& Radford, D. R. (2017). Stress and well-being in dental hygiene and dental therapy students. BDJ Team, 4(8), 17136. https://doi.org/10.1038/bdjteam.2017.136

Hernández, R., Fernández, C., \& Baptista, P. (2010). Metodología de la investigación. Mc Graw Hill.

Jaramillo, G., Caro, H., Parra, Z. A. G., Bedoya, J. P. M., Pabón, É. A. R., \& Mejía, M. C. S. (2008). Dispositivos desencadenantes de estrés y ansiedad en estudiantes de Odontología de la Universidad de Antioquia. Revista Facultad de Odontología, 20(1), 1-20.

Macias, A. B. (2007). El Inventario SISCO del estrés académico. Investigación Educativa Duranguense, (7), 90-93.

Maya Hurtado, C. (2016). Ansiedad y estrés en los estudiantes de odontología. Universidad de Sevilla.

Misrachi-Launert, C., Ríos-Erazo, M., Manríquez-Urbina, J. M., Burgos-Ibarra, C., \& PonceEspinoza, D. (2015). Fuentes de estrés percibidas y rendimiento académico de estudiantes de odontología chilenos. FEM: Revista de La Fundación Educación Médica, 18(2), 109-116. https://doi.org/10.4321/S2014-98322015000200006 
Ortiz-Fernández, L., Moromi- Nakata, H., Quintana del Solar, C., Barra-Hinostroza, M., Bustos de la Cruz, J., Cáceres, L., Chein-Villacampa, S., \& Rodríguez-Vargas, C. (2015). Estrategias, estilos de aprendizaje y rendimiento académico en estudiantes ingresantes de Odontología. Odontología Sanmarquina, 17(2), 65-76. https://doi.org/10.15381/os.v17i2.11050

Pérez, F., \& Cartes, R. (2015). Estrés y burnout en estudiantes de Odontología: una situación preocupante en la formación profesional. Edumecentro, 7(2), 179-190.

Presta, A. A., Saliba Garbin, C. A., Garbin, Í., José, A., Saliba, O., \& Dossi, A. P. (2006). El estrés en la práctica odontológica. Revista de la Asociación Dental Mexicana, 63(5), 185-188.

Radillo, B. E. P., Gómez, S. Z., Velasco, M. A. A., Fernández, M. A., López, T. M. T., \& Franco, M. A. R. (2011). El cirujano dentista ante estrés no percibido como un riesgo ocupacional. Revista cubana de salud y trabajo, 12(1), 10-5.

Reyes Sanjuán, A., Orozco Linero, E. R., Vergara Hernández, C. I., \& Carbonell Muñoz, Z. (2017). Percepción de los estudiantes sobre las estrategias de enseñanza en la Facultad de Odontología [Doctoral dissertation, Universidad de Cartagena].

Sierra, J. C., Ortega, V., \& Zubeidat, I. (2003). Ansiedad, angustia y estrés: tres conceptos a diferenciar. Revista mal-estar e subjetividade, 3(1), 10-59.

Stes, A., De Maeyer, S., \& Van Petegem, P. (2013). Examining the Cross-Cultural Sensitivity of the Revised Two-Factor Study Process Questionnaire (R-SPQ-2F) and Validation of a Dutch Version. PLOS ONE, 8(1), e54099. https://doi.org/10.1371/journal.pone.0054099

Tamayo, J. A., Rodríguez, K., Escobar, K., Mejía, A. M. (2015). Estilos de vida de estudiantes de odontología. Hacia la promoción de la salud, 20(2), 147-160. https://doi.org/10.17151/hpsal.2015.20.2.10

Terán, E., \& Mayta-Tovalino, F. (2019). Risk Factors, Self-perceived Stress, and Clinical Training among Dentistry Students in Peru: A Cross-sectional Study. The Journal of Contemporary Dental Practice, 20(5), 561-565.

Vergara, C. I., \& Navas, M. E. D. (2017). Re-enamorarse de la carrera, una nueva oportunidad de cumplir el sueño de ser odontólogo. Una teoría en Odontología: Transición básico-clínica de los programas de Odontología en las universidades estatales de la Costa Caribe colombiana [Doctoral dissertation, Universidad de Cartagena].

Vergara, C., \& Zaror, C. (2008). Proceso de enseñanza-aprendizaje en la clínica 
odontológica. Aspectos teóricos. Rev Educ Cienc Salud, 5(1), 6-11.

Vergara, K. A., Cárdenas, S. D., \& Martínez, F. G. (2013). Síntomas de depresión, ansiedad y estrés en estudiantes de odontología: prevalencia y factores relacionados. Revista Colombiana de Psiquiatría, 42(2), 173-181.

Willis, G. B., \& Lessler, J. T. (1999). Question appraisal system QAS-99. National Cancer Institute. 